\title{
Commentaire
}

\section{Débarrasser la médecine canadienne des thérapies de conversion}

\author{
Travis Salway PhD, Florence Ashley BCL/JD LLM (bioéthique)
}

ש Citation : CMAJ 2022 January 10;194:E17-8. doi : 10.1503/cmaj.211709-f

Voir la version anglaise de l'article ici : www.cmaj.ca/lookup/doi/10.1503/cmaj.211709

$\mathbf{L}$ 'expression « thérapie de conversion » est un terme générique qui décrit un ensemble mal défini d'interventions psychologiques, comportementales, physiques et confessionnelles qui s'efforcent de supprimer l'attirance envers les personnes de même sexe ou qui dissuadent les patients d'exprimer des identités de genre qui diffèrent du sexe assigné à la naissance ${ }^{1,2}$. Plus de 50 organismes regroupant des professionnels de la santé - dont l'Association des psychiatres du Canada et l'Association médicale américaine - ont émis des déclarations de consensus dénonçant les thérapies de conversion, s'appuyant sur des données probantes empiriques substantielles démontrant que ces pratiques sont inefficaces et liées à de mauvais effets sur la santé, notamment des idées suicidaires et des tentatives de suicide ${ }^{3-7}$. Malgré ces dénonciations, des thérapies de conversion ont toujours cours au Canada, dans des milieux de pratique faisant ou non l'objet d'un permis, touchant jusqu'à $10 \%$ des personnes bispirituelles, lesbiennes, gaies, bisexuelles, transgenres et queers (2SLGBTQ+) ${ }^{8}$. La récente adoption du projet de loi C-4 par le Parlement fédéral interdisant les thérapies de conversion crée une nouvelle occasion de synergie entre les domaines médical et juridique afin de protéger les droits et la santé des personnes 2SLGBTQ+ au Canada.

La caractéristique déterminante des thérapies de conversion et des pratiques comparables ( $p$. ex., les chirurgies d'assignation sexuelle, le refus de soins d'affirmation de genre) est une idéologie qui favorise les identités et les comportements hétérosexuels et cisgenres au détriment de tous les autres. Cette idéologie, qui comprend le cissexisme et l'hétérosexisme, maintient l'hypothèse socionormative que les identités cisgenres et hétérosexuelles sont davantage légitimes que celles des personnes 2 SLGBTQ+ ${ }^{1}$.

Une récente étude empirique réalisée au Canada précise que les thérapies de conversion ne représentent pas une pratique monolithique. Au Canada, comme aux États-Unis, elles ne relèvent pas exclusivement du domaine des praticiens non réglementés ${ }^{2,4}$. Dans une enquête de 2019-2020 comptant 9214 hommes gais, bisexuels, queers et bispirituels, $10 \%$ ont mentionné avoir été exposés aux thérapies de conversion au cours de leur vie, de ce nombre, 30\% avaient vécu cette pratique dans le cabinet d'un professionnel de la santé autorisé ${ }^{8}$. Au cours d'entrevues, des «patients » de thérapies de conversion décrivent avoir participé à des interventions dans un large éventail de lieux : églises, camps, conférences, clavardages en

\section{Points clés}

- Des thérapies de conversion se poursuivent au Canada dans des établissements réglementés et non associés, incitant les gouvernements fédéral, provinciaux, territoriaux et municipaux à débattre et à adopter des dizaines d'interdictions législatives au cours des 6 dernières années.

- Les professionnels de la santé, les patients, les parents, les responsables des orientations politiques et les politiciens ont besoin de renseignements plus précis sur la nature des thérapies de conversion afin de cibler et d'éviter des pratiques comparables qui pourraient causer préjudice aux patients bispirituels, gais, bisexuels, transgenres, queers de même qu'aux patientes lesbiennes (2SLGBTQ+).

- La caractéristique déterminante des thérapies de conversion et des pratiques comparables (p. ex., les chirurgies d'assignation sexuelle, le refus de soins d'affirmation de genre) est une idéologie qui favorise les identités et les comportements hétérosexuels et cisgenres au détriment de tous les autres.

- Les organismes de réglementation du Canada devraient agir afin de renforcer toute nouvelle loi fédérale exprimant clairement qu'ils prendront des mesures disciplinaires immédiates à l'encontre des membres qui causeraient des préjudices en prenant part aux interventions de thérapies de conversion ou en les soutenant.

- Avec l'adoption du projet de loi C-4 et une plus forte gouvernance professionnelle, les médecins du Canada seront doublement incités à rejeter le cissexisme et l'hétérosexisme et à adopter une approche plus inclusive des soins de santé.

ligne, groupes de prières, bureaux de conseillers réglementés et non réglementés de même que dans des cabinets médicaux ${ }^{1}$. Bien que les données contemporaines sur les thérapies de conversion soient limitées par les méthodes d'échantillonnages restreintes requises pour joindre les personnes 2SLGBTQ+ et celles qui ont survécu aux thérapies de conversion, ces données donnent un aperçu de l'ampleur et de la nature du problème auquel nous faisons face. Il n'existe pas de données récentes sur l'attitude des professionnels de la santé autorisés au Canada envers les thérapies de conversion; cependant, une récente publication provenant du Royaume-Uni démontre que plus de $4 \%$ des psychothérapeutes ont traité au moins 1 patient dans le but de réduite l'attirance envers les personnes de même sexe ${ }^{9}$. Les antécédents de professionnels de la 
santé autorisés « traitant » des patients transgenres dans l'optique d'harmoniser leur identité de genre avec le sexe assigné à la naissance sont probablement beaucoup plus prévalents ${ }^{4,8}$. Des données provenant de l'enquête Trans PULSE Canada de 2019 regroupant 2033 personnes indiquent que parmi les répondants transgenres et non binaires qui ont subi une thérapie de conversion, $27 \%$ ont rapporté que cette pratique impliquait un médecin, un membre du personnel infirmier ou les deux (Ayden Scheim, Université Drexel, Philadelphie (Pennsylvanie); communication personnelle, 2021). Les données portant sur les formes que prennent ces pratiques sont hétérogènes; bien que certains personnes interrogées au Canada ont divulgué des pratiques physiques graves, comme la thérapie électroconvulsive, la plupart ont décrit des psychothérapies, chacune avec un parti pris explicite ou implicite à l'encontre d'un résultat qui pourrait comprendre une identité 2 SLGBTQ+1,7.

Les autorités de réglementation médicale du Canada devraient agir afin d'augmenter la portée des lois fédérales en exprimant clairement qu'elles prendront des mesures disciplinaires immédiates à l'encontre des membres qui causent des préjudices en participant ou soutenant des interventions de thérapies de conversion. Bien que plusieurs provinces et territoires (p. ex., l'Ontario, la Nouvelle-Écosse et le Yukon) aient adopté des lois interdisant l'emploi de fonds publics à la pratique de thérapies de conversion, la manière dont ces lois sont appliquées n'est pas claire. Des stratégies de consolidation mutuelles pour dissuader le recours à des pratiques de thérapie de conversion - dont des actions législatives et réglementaires - sont probablement requises en raison des formes insidieuses et cachées que ces pratiques prennent souvent ${ }^{1}$. La population canadienne a le droit d'obtenir des soins médicaux soutenant les identités 2SLGBTQ+. Par conséquent, les médecins qui ne sont pas familiers avec une pratique médicale d'affirmation de genre, ou qui ne sont pas à l'aise de prodiguer de tels services, ont la responsabilité d'orienter leurs patients vers des médecins qui peuvent offrir ces services ${ }^{10}$.

En ciblant la caractéristique déterminante des thérapies de conversion comme une idéologie sous-jacente qui favorise les identités et les comportements hétérosexuels et cisgenres au détriment de tous les autres, les professionnels de la santé, les usagers et les responsables des orientations politiques peuvent adopter des politiques plus précises et plus efficaces afin d'atteindre une équité en matière de santé sexuelle et de genre au Canada. Avec l'adoption du projet de loi C-4 et une plus forte gouvernance professionnelle, les médecins du Canada seront doublement incités à rejeter le cissexisme et l'hétérosexisme et à adopter une approche plus inclusive des soins de santé. Les ingrédients sont en place pour débarrasser notre système médical des thérapies de conversion, une fois pour toutes.

\section{Références}

1. Kinitz DJ, Goodyear T, Dromer E, et al. "Conversion therapy" experiences in their social contexts: a qualitative study of sexual orientation and gender identity and expression change efforts in Canada: Les expériences de "thérapie de conversion" dans leur contexte social: une étude qualitative. Can J Psychiatry 2021 July 9 [Cyberpublication avant l'impression];7067437211030498. doi: 10.1177/07067437211030498.

2. Streed CG, Anderson JS, Babits C, et al. Changing medical practice, not patients - putting an end to conversion therapy. N Engl J Med 2019;381:500-2.

3. Salway T, Ferlatte $O$, Gesink D, et al. Prevalence of exposure to sexual orientation change efforts and associated sociodemographic characteristics and psychosocial health outcomes among Canadian sexual minority men. Can J Psychiatry 2020;65:502-9.

4. Turban JL, Beckwith N, Reisner SL, et al. Association between recalled exposure to gender identity conversion efforts and psychological distress and suicide attempts among transgender adults. JAMA Psychiatry 2020;77:68-76.

5. Ashley F. Model law - prohibiting conversion practices. SSRN 2019 May 28. Accessible ici : https://papers.ssrn.com/sol3/papers.cfm?abstract_id=3398402 (consulté le 2 oct. 2021).

6. Blosnich JR, Henderson ER, Coulter RWS, et al. Sexual orientation change efforts, adverse childhood experiences, and suicide ideation and attempt among sexual minority adults, United States, 2016-2018. Am J Public Health 2020; May 21;110(7) [Cyberpublication avant l'impression]:e1-e7. doi: 10.2105/AJPH.2020.305637.

7. Goodyear T, Kinitz DJ, Dromer E, et al. "They want you to kill your inner queer but somehow leave the human alive": delineating the impacts of sexual orientation and gender identity and expression change efforts. J Sex Res 2021 Apr. 19 [Cyberpublication avant l'impression];1-11. doi: 10.1080/00224499.2021.1910616.

8. Salway T, Juwono S, Klassen B, et al. Experiences with sexual orientation and gender identity conversion therapy practices among sexual minority men in Canada, 2019-2020. PLoS One 2021;16:e0252539.

9. King M. Attitudes of therapists and other health professionals towards their LGB patients. Int Rev Psychiatry 2015;27:396-404.

10. Lam JSH, Abramovich A. Transgender-inclusive care. CMAJ 2019;191:E79.
Intérêts concurrents : Travis Salway mentionne recevoir des subventions des Instituts de recherche en santé du Canada, du Conseil de recherches en sciences humaines du Canada, de l'Initiative de recherche engagée auprès de la collectivité de l'Université SimonFraser, de l'organisme à but non lucratif SUPPORT Unit de la Colombie-Britannique et de la Fondation Michael-Smith pour la recherche en santé. Travis Salway est soutenu par une bourse de la Fondation Michael-Smith pour la recherche en santé. Florence Ashley est soutenue par une bourse d'étude doctorale Joseph-Armand-Bombadier du Conseil de recherches en sciences humaines. Les deux auteurs travaillent en étroite collaboration avec des organismes communautaires qui regroupent des personnes bispirituelles, lesbiennes, gaies, bisexuelles, transgenres et queers afin de cibler les politiques et les interventions qui soutiennent l'équité en matière de santé pour ces communautés. Aucun autre intérêt concurrent n'a été déclaré.

Cet article a été révisé par des pairs.

Affiliations: Faculté des sciences de la santé (Salway), Université Simon Fraser, Burnaby BC; Centre de contrôle des maladies de la Colombie-Britannique (Salway); Centre pour l'équité en matière de santé sexuelle et de genre (Salway), Vancouver, BC; Faculté de droit et Centre conjoint de bioéthique de l'Université de Toronto (Ashley), Toronto, Ont.

Collaborateurs : Travis Salway a produit l'ébauche du manuscrit. Les deux auteurs ont contribué à la conception du travail, ont révisé de façon critique le contenu intellectuel important du manuscrit, ont donné leur approbation finale pour la version destinée à être publiée et assument l'entière responsabilité de tous les aspects du travail.
Propriété intellectuelle du contenu : Il s'agit d'un article en libre accès distribué conformément aux modalités de la licence Creative Commons Attribution (CC BY-NC-ND 4.0), qui permet l'utilisation, la diffusion et la reproduction dans tout médium à la condition que la publication originale soit adéquatement citée, que l'utilisation se fasse à des fins non commerciales (c.-à-d., recherche ou éducation) et qu'aucune modification ni adaptation n'y soit apportée. Voir : https://creativecommons.org/ licenses/by-nc-nd/4.0/deed.fr

Remerciements : Les auteurs remercient les courageux survivants des thérapies de conversion qui ont raconté sans relâche leurs histoires afin que le reste d'entre nous puisse finalement passer à l'acte pour combattre ces pratiques traumatisantes.

Correspondance : Travis Salway, travis_salway@sfu.ca 